\section{Kniearthrose: Adipöse Patienten bleiben auch nach Gewichtsabnahme bewegungsarm}

Bartholdy C et al. Association Between Weight Loss and Spontaneous Changes in Physical Inactivity in Overweight/Obese Individuals With Knee Osteoarthritis: An Eight-Week Prospective Cohort Study. Arthritis Care Res (Hoboken) 2020; 72: 397-404. doi: 10.1002/acr.23868

Adipöse Menschen, die gleichzeitig an einer Kniegelenkarthrose leiden, meiden in der Regel körperliche Belastungen. Angesichts der mit einem inaktiven Lebensstil verbundenen Risiken - es drohen bspw. Herzerkrankungen, ein Diabetes mellitus sowie ein frühzeitiger Tod - müssen die Betroffenen zu ausreichend Bewegung motiviert werden. Nimmt nach einer diätbedingten $\mathrm{Ge}$ wichtsabnahme ihre Aktivität zu?

Diese Fragestellung untersuchten dänische Wissenschaftler im Rahmen einer prospektiven Kohortenstudie. Teilnehmer waren 124 adipöse Personen (Bodymassindex $\geq 27 \mathrm{~kg} / \mathrm{m}^{2}$ ) mit einer symptomatischen Kniegelenkarthrose. Ein geplanter bariatrischer oder Knieeingriff sowie aktuell andauernde medikamentöse oder diätetische Maßnahmen zur Gewichtsreduktion stellten Ausschlusskriterien dar. Alle Probanden absolvierten über 8 Wochen eine intensive Diätintervention mit dem Ziel der Gewichtsabnahme. Diese umfassten den Ersatz der Mahlzeiten durch spezielle Produkte (tägliche Energiezufuhr 800-1000 kcal) sowie von Ernährungswissenschaftlern angeleitete Schulungen. Die körperliche Aktivität der Probanden objektivierten die Wissenschaftler mithilfe eines 24 Stunden pro Tag an der Hüfte getragenen Akzelerometers. Anhand dieser Messdaten prüften sie, inwiefern sich im Verlauf des Studienzeitraums (eine Woche vor Beginn der Diät bis zum Ende der achtwöchigen Intervention) der durchschnittliche Anteil der pro Tag in körperlicher Inaktivität verbrachten Zeit (sitzend, ruhend, schlafend) veränderte.

\section{Ergebnisse}

Im Schnitt verloren die Studienteilnehmer im Verlauf der achtwöchigen Interventionsphase 12,7 kg Körpergewicht, was einer Abnahme des Bodymassindex um 4,3 kg/m² entsprach. Die mittels Knee Injury and Osteoarthritis Outcome Score objektivierten arthrotischen Kniebeschwerden besserten sich ebenfalls in signifikantem und klinisch relevantem Umfang. Trotz dieser Effekte beobachteten die Wissenschaftler keine wesentliche Veränderung der Bewegungsmuster: Die Probanden verbrachten vor und nach der Intervention pro Tag ähnlich lange Zeit in körperlicher Inaktivität (durchschnittliche Veränderung: 8,8 Min. pro Tag; $95 \% \mathrm{KI}-12,1$ bis 29,$7 ; \mathrm{p}=0,41$ ).

\section{FAZIT}

Adipöse Kniearthrosepatienten behalten trotz Gewichtsabnahme und klinisch relevanter Verringerung der Gelenksymptomatik ihre Bewegungsgewohnheiten bei und bleiben unverändert inaktiv, schlussfolgern die Wissenschaftler. Da spontane Verhaltensänderungen offenbar nicht eintreten, müssen diese gezielt stimuliert werden: Beispielsweise durch Aufklärung über die Folgen von Bewegungsarmut. Nur so könne den bekannten Gesundheitsrisiken vorgebeugt und eine Stabilisierung des Körpergewichts erreicht werden.

Dr. med. Judith Lorenz, Künzell 